\title{
Défis liés à l'élimination durable de la rougeole au Canada
}

\author{
Crowcroft N. S. ${ }^{1,2,3^{*}}$ \\ 1 Santé publique Ontario, Toronto (Ontario) \\ 2 Département de médecine de laboratoire et de biopathologie, Université de Toronto, Toronto (Ontario) \\ 3 Dalla Lana School of Public Health, Université de Toronto, Toronto (Ontario) \\ * Auteure-ressource : Natasha.crowcroft@oahpp.ca
}

Les récentes importations de rougeole au Canada n'ont généralement pas conduit à de grandes éclosions, ce qui indique que la maladie est bien contrôlée au Canada. Les grandes éclosions isolées qui ont eu lieu nous rappellent qu'il faut rester vigilant. La rougeole présente des défis particuliers, car c'est la maladie la plus contagieuse connue. Elle se développe chez les personnes qui n'ont pas accès au système de santé infantile pour une raison ou une autre, et nous ne disposons pas toujours de l'information nécessaire pour déterminer et cibler les collectivités à faible taux de vaccination. Les éclosions sont généralement dues à des Canadiens qui voyagent à l'étranger et sont exposés à la rougeole. La lutte contre les éclosions sporadiques résultant de l'importation du virus exige beaucoup de temps et de ressources, ce qui rend prioritaire la vaccination pour les Canadiens voyageant à l'extérieur de la Région des Amériques (où la rougeole a été éliminée). Pour éviter tout risque d'importation de la rougeole au Canada, les autres pays et régions du monde doivent faire des progrès dans l'élimination de la maladie.

Les récentes importations de rougeole au Canada nous rappellent en fait l'incroyable réussite de la vaccination dans l'élimination de cette maladie. Cela tient au fait que, à quelques exceptions près, la majorité des importations n'ont pas conduit à d'autres cas ou n'ont causé que de petites éclosions, ce qui indique que, dans l'ensemble, la rougeole est actuellement bien contrôlée au Canada (1). La taille des éclosions (y compris les cas où il n'y a pas eu de retransmission) peut servir à estimer le niveau de contrôle en calculant le taux de reproduction effectif $(\mathrm{Re})$, qui est défini comme le nombre moyen de personnes réellement infectées par chaque cas pendant une période donnée dans une population avec un certain taux d'immunité (2). Les provinces comme l'Ontario, où un seul cas est défini comme une éclosion, peuvent calculer le taux Re. Une analyse des données provenant de 13 éclosions en décembre 2009 a révélé un taux Re estimé de 0,52, soit un taux bien en dessous du seuil épidémique où $\operatorname{Re}=1$ (3).

Les récentes éclosions de rougeole au Canada incluaient des cas typiques caractérisés par de la fièvre, de la toux et une éruption cutanée maculopapulaire. Les patients ont été hospitalisés, mais, heureusement, il n'y a eu aucun décès depuis que nous avons obtenu le statut d'élimination en 1997. En 2011, 10 décès dus à la rougeole sont survenus en France. Cette année-là, les épidémies de rougeole ont explosé dans toute l'Europe, avec plus de 30000 cas signalés au Centre européen pour la prévention et le contrôle des maladies $(4,5)$. Après des importations répétées dues à l'épidémie de 2011 en Europe, le Québec a connu la plus grande éclosion de rougeole de tous les pays de l'Amérique du Nord, de l'Amérique du Sud et de l'Amérique centrale depuis 2001, en enregistrant au total 776 cas entre 2011 et 2012. Cela a menacé le statut d'élimination de toute la région (6). L'éclosion était principalement due à un taux de vaccination inférieur à ce qui était nécessaire pour l'élimination (6), ce qui explique pourquoi les compétences ne peuvent pas se montrer complaisantes et ont besoin de données de haute qualité sur le taux de vaccination jusqu'à l'échelle des districts et pour tous les groupes d'âge, afin de déterminer les zones à risque et de prendre des mesures efficaces lorsque des lacunes d'immunité sont décelées.

Nous savons qu'il existe des lacunes d'immunité dans les collectivités qui rejettent la vaccination ou dans les zones où le taux de vaccination n'est tout simplement pas assez élevé. Les questions qui se posent surle taux exact de vaccination et le taux d'immunité de la population ne trouveront pas de réponse en l'absence d'un registre des vaccinations ou d'une surveillance sérologique. Le fait que les trois quarts des cas en 2013 n'avaient pas été vaccinés laisse supposer que le taux de vaccination est plus faible que nous le pensons, car nous pourrions nous attendre à ce que la plupart des cas soient vaccinés si le taux de vaccination était élevé. 
La rougeole présente un défi particulier, puisque c'est la maladie la plus contagieuse connue, avec un taux de reproduction de base de l'ordre de 17 (ce qui signifie que dans une population entièrement à risque, chaque personne infectée infecterait à son tour, en moyenne, 17 autres). L'immunité de la population de plus de $95 \%$ est donc nécessaire pour l'élimination (7). En tenant compte des échecs vaccinaux, cela signifie que notre système doit atteindre un taux de vaccination par deux doses de $97 \%$ pour appuyer l'élimination, ce qui implique un contrôle rigoureux du système de santé infantile. On ne peut pas se cacher de la rougeole et, tôt ou tard, le virus trouvera une personne à risque. Cela a été bien démontré lors de l'éclosion de rougeole de 2013 en Alberta, dans le cadre de laquelle chaque personne exposée qui n'était pas vaccinée ou n'avait pas d'antécédent de rougeole a été infectée (8). Les éclosions qui ont eu lieu ces dernières années illustrent pourquoi le taux de vaccination contre la rougeole doit être à la fois élevé et uniforme et comment les personnes à risque se regroupent de façon non aléatoire. L'évaluation de la vaccination dans les écoles et les collectivités révèle, comme en témoigne le Sud de l'Alberta, les variations et les lacunes dans l'immunité et permet à la santé publique d'élaborer des stratégies ciblées pour améliorer le taux de vaccination et d'établir des plans en cas d'éclosion (9).

La rougeole met en lumière ceux qui, pour une raison ou une autre, n'ont pas accès au système de santé infantile, y compris les groupes religieux qui refusent la vaccination, les enfants dont les parents hésitent à les faire vacciner et les groupes marginalisés tels que les peuples autochtones. Les indicateurs relatifs aux programmes de vaccination contre la rougeole (p. ex. le taux de vaccination et de mortalité due à la rougeole) servent d'indicateurs de la qualité du système de santé infantile concernant l'accès aux soins primaires et de base de santé publique (p. ex. dans le cadre des objectifs du Millénaire pour le développement). Cette approche peut être utilisée pour comparer le Canada avec d'autres pays, comme dans le rapport sur le bien-être de l'enfant et de la mère des Fonds des Nations Unies pour l'enfance, dans lequel le Canada se classait au $28^{\mathrm{e}}$ rang sur 29 pays en raison de son taux de vaccination (10). Il est clair que des améliorations peuvent encore être apportées. Des données de très haute qualité sont nécessaires à petite échelle afin d'être en mesure de démontrer que nous assurons le contrôle. Les méthodes utilisées dans l'Enquête nationale sur la vaccination qui consistent à joindre un échantillon de la population par téléphone peuvent se révéler insuffisantes à cet effet, car les répondants peuvent ne pas être représentatifs de la population générale ou suffisamment nombreux pour représenter les petites collectivités. Même petites, les collectivités qui n'ont pas eu accès à la vaccination ou l'ont refusée restent assez grandes pour atteindre la taille critique de la collectivité (de 250000 à 400000 individus) nécessaire pour assurer la transmission de la rougeole (11). Les communautés religieuses qui n'acceptent pas la vaccination peuvent être exposées à la rougeole en raison de leurs liens étroits avec les régions où la rougeole n'a pas été éliminée. Nous pouvons tirer des leçons des approches adoptées aux PaysBas, où les données sur l'immunité locale ont servi à étudier les répercussions de l'hétérogénéité de la vaccination dans les communautés religieuses liées à celles du Canada et permis de prédire la grande éclosion qui a émergé aux Pays-Bas en 2013 (12,13).

Plutôt que d'être stigmatisés comme source possible de risque, les immigrants au Canada peuvent en fait jouer un rôle important dans la réduction des risques de rougeole dans ce pays. Près de $20 \%$ des Canadiens sont nés à l'étranger et, comme la plupart sont originaires de pays où la rougeole est endémique, ils seraient arrivés ici vaccinés, après avoir eu la rougeole pendant leur enfance. En tant que citoyens, ils sont aussi plus susceptibles d'être immunisés (14). De plus, la plupart des cas importés concernaient des Canadiens ayant voyagé à l'étranger et ayant rapporté la rougeole plutôt que des visiteurs au Canada (1). II s'agit d'une bonne nouvelle, puisque les Canadiens devraient être plus faciles à joindre que les visiteurs, afin de s'assurer que leurs enfants et eux-mêmes sont suffisamment protégés contre la rougeole avant de voyager.

Des importations sont attendues aussi longtemps que la rougeole continue de circuler dans d'autres régions du monde. Assurer l'élimination de la rougeole en cas d'importation est un processus long et coûteux, qui comprend l'important travail de suivi des cas et des contacts ainsi que le contrôle des éclosions. II n'existe aucun chiffre disponible pour le Canada, mais aux États-Unis, le coût de la réponse aux éclosions de rougeole en 2011 pour les services de santé publique allait de 2,7 à 5,3 millions de dollars américains (15). Dans le cadre de ce travail, la combinaison d'une bonne microbiologie et d'une bonne épidémiologie de la part de la santé publique est la définition d'une surveillance et d'une réponse de haute qualité en cas d'éclosion de la rougeole. Le Laboratoire national de microbiologie de l'Agence de la santé publique du Canada joue un rôle de 
premier plan en procédant au génotypage des cas de rougeole et est bien placé pour fournir le séquençage du génome entier qui sera nécessaire pour produire une preuve moléculaire de l'origine des cas (16).

Les partenariats internationaux sont essentiels pour assurer l'élimination de la rougeole, y compris la liaison avec l'OPS (branche régionale de l'OMS). Dans les pays fédéraux très décentralisés, comme le Canada, les rôles de la santé publique sont partagés entre plusieurs ordres de gouvernement. Cela peut constituer un obstacle pour des partenaires internationaux comme l'OPS. L'Agence de la santé publique du Canada est responsable des services de laboratoire de référence et doit présenter des rapports hebdomadaires à l'OPS, mais les provinces et les territoires travaillent avec les organismes locaux de santé publique pour surveiller les éclosions et y répondre. Le Canada peut aider en montrant au monde entier la façon dont tous les niveaux responsables de la santé publique peuvent travailler ensemble de façon transparente et avec succès. Les États-Unis ont appuyé les efforts en vue d'éliminer la rougeole dans la Région des Amériques pour réduire le fardeau que représente la rougeole importée et reconnaissent la valeur pour les Américains de la lutte contre la rougeole dans d'autres régions (17). Maintenant que la rougeole est essentiellement importée d'autres régions, le temps est peut-être venu pour le Canada d'assurer non seulement l'élimination de la maladie dans ses propres frontières, mais aussi de travailler avec l'OPS dans notre région et l'OMS à l'échelle mondiale pour soutenir d'autres régions du monde dans l'élimination de la rougeole. En luttant contre la rougeole, le Canada s'acquitte de ses obligations internationales. Toutefois, les importations ne cesseront pas jusqu'à ce que la rougeole soit éliminée dans tous les pays du monde. Au bout du compte, cela permettra d'éviter toute importation de la maladie, de réduire les coûts et de sauver des vies dans le monde entier.

\section{Références}

(1) Shane, A., Hiebert, J., Sherrard, L., Deehan, H. La surveillance de la rougeole au Canada : Tendances 2013. Relevé des maladies transmissibles au Canada, vol. 40-12, 2014;244.

(2) De Serres G, Gay NJ, Farrington CP. Epidemiology of transmissible diseases after elimination. Am J Epidemiol 2000; 151:1039-1048. http://aje.oxfordjournals.org/content/151/11/1039

(3) Lim, G.H., Deeks, S.L., Fediurek, J., Gubbay, J., Crowcroft, N.S. Consignation de l'élimination de la rougeole, de la rubéole et de l'embryopathie rubéolique en Ontario : 2009-2012. Relevé des maladies transmissibles au Canada, vol. 40-8, 2014.

(4) Number of cases of measles reported to The European Surveillance System (TESSy) by EU and EEA countries, 2011. Accès : http://www.ecdc.europa.eu/en/healthtopics/measles/epidemiological_data/Pages/Numberof-measles-cases-2011.aspx.

(5) Antona D, Levy-Bruhl D, Baudon C, Freymuth F., Lamy M, Maine C, et al. Measles elimination efforts and 2008-2011 outbreak, France. Emerg Infect Dis. 2013; 19(3):357-64.

(6) De Serres G, Markowski F, Toth E, Landry M, Auger D, Mercier M, et al. Largest measles epidemic in North America in a decade--Quebec, Canada, 2011: contributions of susceptibility, serendipity, and superseeding events. J Infect Dis. 2013; 207(6):990-8.

(7) Gay NJ, Hesketh LM, Morgan-Capner P, Miller E. Interpretation of serological surveillance data for measles using mathematical models: implications for vaccine strategy. Epidemiol Infect. 1995; 115(1):139-56.

(8) Kershaw, T., Suttorp, V., Simmonds, K., St. Jean, T. Éclosions de rougeole au sein d'une population ne pratiquement pas la vaccination, Alberta, 2013. Octobre-novembre 2013. Relevé des maladies transmissibles au Canada, vol. 40-12, 2014;270.

(9) Matkin, A., Simmonds, K., Suttorp, V. Taux d'administration du vaccin contenant le virus de la rougeole dans le Sud de l'Alberta. Relevé des maladies transmissibles au Canada, vol. 40-12, 2014;263.

(10) Coincé au milieu. Le bien-être des enfants dans les pays riches : Vue d'ensemble comparative. Unicef Canada. http://www.unicef.ca/sites/default/files/imce_uploads/DISCOVER/OUR\%20WORK/ADVOCACY/DO MESTIC/POLICY\%20ADVOCACY/DOCS/unicef_bilan_innocenti_11_document_accompagnement_canada.pdf 
(11) Keeling MJ, Grenfell BT. Disease extinction and community size: modeling the persistence of measles. Science. 1997; 275(5296):65-7.

(12) Glass K, Kappey J, Grenfell BT. The effect of heterogeneity in measles vaccination on population immunity. Epidemiol Infect. 2004; 132:675-683.

(13) Mollema L, Smits GP, Berbers GA, Van Der Klis FR, Van Binnendijk RS, De Melker HE, Hahné SJ. High risk of a large measles outbreak despite 30 years of measles vaccination in The Netherlands. Epidemiol Infect. May 2014; 142(5):1100-8.

(14) Quach S, Hamid JS, Pereira JA, Heidebrecht CL, Deeks SL, Crowcroft NS, Quan SD, Brien S, Kwong JC. Influenza vaccination coverage across ethnic groups in Canada. Public Health Agency of Canada/Canadian Institutes of Health Research Influenza Research Network Vaccine Coverage Theme Group. CMAJ. Oct 16 2012; 184(15):1673-81. doi: 10.1503/cmaj.111628.

(15) Ortega-Sanchez IR1, Vijayaraghavan M2, Barskey AE2, Wallace GS2Vaccine. 2014 Mar 5; 32(11):1311-7. doi: 10.1016/j.vaccine.2013.10.012. Epub 2013 Oct 14. The economic burden of sixteen measles outbreaks on United States public health departments in 2011.

(16) Hiebert, J., Severini, A. Épidémiologie moléculaire de la rougeole : Quelle information nous apporte-t-elle et pourquoi est-ce important? Relevé des maladies transmissibles au Canada, vol. 40-12, 2014;286.

(17) CDC. Manual for the Surveillance of Vaccine-Preventable Diseases. Chapter 7 Measles.

Accès : http://www.cdc.gov/vaccines/pubs/surv-manual/chpt07-measles.html . 